\title{
The involvement of cell cycle events in the pathogenesis of Alzheimer's disease
}

Karl Herrup*

\begin{abstract}
Most neurons undergo their last cell division within the first 1 to $2 \%$ of the lifespan of an organism. This has been interpreted to mean that adult neurons are permanently postmitotic, but Alzheimer's disease (AD) is an example of a late-onset neurodegenerative disease that challenges this concept. In AD, neurons in populations at risk for death reactivate their cell cycle and replicate their genome - but rather than complete the cycle with mitosis and cytokinesis, the neurons die. While opening new perspectives on the etiology of AD dementia, the simple linear model suggested by this description gains in complexity with the maturation of the adult brain. This complexity makes the full understanding of the relationship between cell division and cell death more difficult to achieve. The quest for understanding is worthwhile, however, as fresh avenues for therapeutic intervention are the prizes for success.
\end{abstract}

For most central nervous system neurons, emigration from the ventricular zone marks their entrance into a state from which they will never normally re-enter a cell cycle. The prohibition against cell division in this new state of existence is sufficiently strong that if a neuron does try to divide then it will die.

The molecular mechanisms that link cell cycle to cell death in this situation are not fully understood, but the relationship is completely straightforward in young postmitotic neurons. As documented in several laboratories, if a neuron is forced to divide by expression of an oncogene, it will re-enter a cycle and die. If any of several cell cycle suppressor functions are lost, the neuron will re-enter a cycle and die. If a cultured neuron is exposed to oxidative stress, DNA damaging agents or certain

*Correspondence: herrup@biology.rutgers.edu

Department of Cell Biology and Neuroscience, Nelson Biological Laboratories, Rutgers University, 604 Allison Road, Piscataway, NJ 08854, USA neurotoxins (including $\mathrm{A} \beta$ aggregates), it will re-enter a cycle and die. The cell cycle events are directly involved in the mechanics of cell death. We know this because if progression through the cycle is blocked, cell cyclerelated neuronal death (CRND) is also blocked. Finally, the developing nervous system itself makes use of CRND during the process of numerical matching. Hours before they die, target-deprived neurons reexpress cell cycle proteins and engage in a new round of DNA synthesis [1-6].

These data lend themselves to a simple linear model of neuronal death as illustrated in Figure 1. The relevance of this model to Alzheimer's disease (AD) is high, underscored by an entire body of evidence that a CRND-like process occurs in the populations of neurons that are lost during the disease - hippocampal formation, frontal cortex, basal nucleus, dorsal raphe and locus coeruleus. In autopsy samples, neuronal cell cycle events are clearly present, detected primarily as re-expression of immunoreactive proteins normally found only in dividing cells. Beyond the immunocytochemical evidence, several laboratories have used cytogenetic techniques to show that the chromosome copy number increases. This means that CRND involves true DNA replication, as distinct from DNA repair.

The use of human autopsy samples places limits on researchers' ability to fully test the cell cycle model in AD, but several qualitative features of the observed neuronal cell cycle activity fit well with the model outlined above. First, every population of neuron that is lost during AD has neurons that stain positive for cell cycle proteins. Second, neuronal populations that are spared in AD are negative for cell cycle activity. Third, cell cycle events have been reported in subjects whose death occurred when a mild cognitive impairment had only recently been detected [7]. This is evidence that the cell cycle events are linked to neuronal cell death at all stages of the disease and do not occur only as co-morbid problems unique to the final stages of the disease process. Fourth, neurons in agematched controls have, on average, an order of magnitude fewer cell cycle-positive neurons. Fifth, in mouse models of $\mathrm{AD}$, cell cycle events accurately recapitulate both the spatial and temporal progression of neuronal loss seen in 


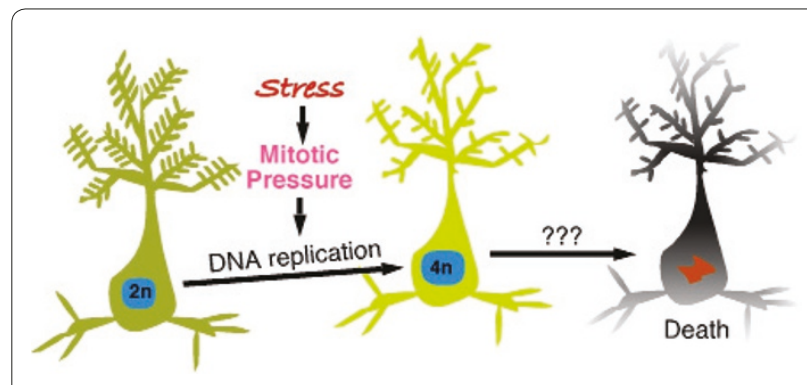

Figure 1. Simple linear model of neuronal death. A simple linear pathway connecting stress transduced through an increase in the probability of entering a cell cycle to DNA replication and, in the embryo, directly to neuronal death. In the mature adult neuron, the linkage to cell death is interrupted (question marks) and the cell can maintain a hyperploid genetic content and survive for months if not years.

human AD. Finally, the linkage of neurodegeneration to ectopic cell cycle reactivation has been documented in diseases apart from $\mathrm{AD}$ - such as ataxia-telangiectasia, Parkinson's disease, stroke, amyotrophic lateral sclerosis, and others.

The strength of this evidence notwithstanding, caution is still advised before applying a simple linear cell cycle model to explain the death of neurons in AD. Viewed objectively, the human data are purely correlative. Rigorous proof of causality (cell cycle $\rightarrow$ cell death) is still missing, and we should therefore move slowly in applying the complete embryonic model to the neurons of the adult brain.

To completely establish the mechanistic role of cell cycle events in $\mathrm{AD}$, two important pieces of data are needed. The first is proof that it is the cycling cells which die. We believe this is true and the experimental data from the embryonic neurons suggest this belief is well founded. But the available evidence does not allow us to disprove the alternative that, in the adult brain, cell cycle re-entrance is protective rather than destructive. The second piece of data needed to establish causality is to show that if we block the cell cycle in $\mathrm{AD}$ neurons then we can block their death. This will be an exceedingly difficult proof to obtain, but a positive outcome would open a fresh approach to disease prevention. The task is therefore worth engaging.

In this vein, an encouraging result has recently been reported in experiments designed to measure the effects of chronic nonsteroidal anti-inflammatory drug (NSAID) administration on the appearance of cell cycle events in an APP transgenic mouse line [8]. In untreated transgenic mice, evidence of neuronal cell cycling occurs in a temporal and spatial pattern that mimics the neurodegeneration found in AD. When either ibuprofen or naproxen is administered before the cycles begin, however, the appearance of these cell cycle events is blocked. This is reminiscent of reports from human epidemiological studies citing the reduced risk of AD in individuals with a history of long-term use of certain NSAIDs [9].

A major unanswered question, given the mouse data, is why prospective NSAID clinical trials in humans have all ended with no evidence of significant effect on $A D$ symptoms. It may be hoped that the discrepancy is explained by the fact that the mouse studies and the epidemiology measure prevention (drug administration is initiated before the cell cycle events began) while the clinical trials measure therapeutic potential (drug treatment begins after the subjects have been identified as having disease). Evidence in support of this idea comes from the mouse studies themselves [8]. When drug administration was delayed until later in the disease process, NSAID treatment was found not to reverse the cell cycle events in neurons in which disease had begun. While speculative, this explanation should keep alive the hope that NSAIDs may yet point the way to effective disease deterrents in humans - even if curative therapies will require other strategies.

\section{Abbreviations}

AD, Alzheimer's disease; CRND, cell cycle-related neuronal death; NSAID, nonsteroidal anti-inflammatory drug.

\section{Competing interests}

The author declares that he has no competing interests.

\section{Published: 20 May 2010}

\section{References}

1. Greene LA, Biswas SC, Liu DX: Cell cycle molecules and vertebrate neuron death: E2F at the hub. Cell Death Differ 2004, 11:49-60.

2. Herrup K, Yang Y: Cell cycle regulation in the postmitotic neuron: oxymoron or new biology? Nat Rev Neurosci 2007, 8:368-378.

3. Webber KM, Casadesus G, Zhu X, Obrenovich ME, Atwood CS, Perry G, Bowen $\mathrm{RL}$, Smith MA: The cell cycle and hormonal fluxes in Alzheimer disease: a novel therapeutic target. Curr Pharm Des 2006, 12:691-697.

4. Arendt T: Synaptic degeneration in Alzheimer's disease. Acta Neuropathol 2009, 118:167-179.

5. Park KH, Hallows JL, Chakrabarty P, Davies P, Vincent I: Conditional neuronal simian virus $40 \mathrm{~T}$ antigen expression induces Alzheimer-like tau and amyloid pathology in mice. J Neurosci 2007, 27:2969-2978.

6. Copani A, Guccione S, Giurato L, Caraci F, Calafiore M, Sortino MA, Nicoletti F: The cell cycle molecules behind neurodegeneration in Alzheimer's disease: perspectives for drug development. Curr Med Chem 2008 , 15:2420-2432.

7. Yang Y, Mufson EJ, Herrup K: Neuronal cell death is preceded by cell cycle events at all stages of Alzheimer's disease. J Neurosci 2003, 23:2557-2563.

8. Varvel NH, Bhaskar K, Kounnas MZ, Wagner SL, Yang Y, Lamb BT, Herrup K: NSAIDs prevent, but do not reverse, neuronal cell cycle reentry in a mouse model of Alzheimer disease. J Clin Invest 2009, 119:3692-3702.

9. Vlad SC, Miller DR, Kowall NW, Felson DT: Protective effects of NSAIDs on the development of Alzheimer disease. Neurology 2008, 70:1672-1677.

doi:10.1186/alzrt37

Cite this article as: Herrup K: The involvement of cell cycle events in the pathogenesis of Alzheimer's disease. Alzheimer's Research \& Therapy 2010, 2:13. 